\title{
Genetic Studies of IgA Nephropathy
}

\author{
Patrick H. Maxwell ${ }^{a}$ Yiming Wang ${ }^{b}$ \\ ${ }^{a}$ Renal Section, Hammersmith Hospital, Imperial College, London, UK; ${ }^{b}$ Department of Medical Genetics, \\ Zhongshan Medical College, Guangzhou, PR China
}

\section{Key Words}

$\lg$ A nephropathy · Glomerulonephritis · Genetics

\begin{abstract}
$\lg$ A nephropathy is the commonest form of glomerulonephritis worldwide, but we still know relatively little about its pathogenesis. Potentially, genetic studies might provide new insights and suggest novel therapeutic approaches to this important cause of chronic kidney disease. Two approaches that are likely to yield new information are analysis of multiply affected pedigrees and large-scale, well-controlled association studies.
\end{abstract}

Copyright @ 2006 S. Karger AG, Basel

IgA nephropathy is a common cause of glomerulonephritis and of end-stage renal failure [1]. Despite much research, many key challenges remain concerning IgA nephropathy, including the following. First, little is known about why certain individuals develop this condition. Second, it is not clear why $20-30 \%$ of patients with IgA nephropathy develop progressive disease, while the others do not. Third, despite the frequency of the condition, effective treatment strategies are limited.

The authors apologise for limited referencing of the background literature owing to the restricted space available.

\begin{tabular}{ll}
\hline KARGER & ( 2006 S. Karger AG, Basel \\
1660-2129/06/1024-0076\$23.50/0 \\
Fax +4161306 1234 \\
$\begin{array}{l}\text { E-Mail karger@karger.ch } \\
\text { www.karger.com }\end{array}$ & $\begin{array}{l}\text { Accessible online at: } \\
\text { www.karger.com/nee }\end{array}$
\end{tabular}

Genetic approaches have achieved substantial insights into many human diseases - most clearly in the case of monogenic diseases with high penetrance. This minireview assesses what we know about genetic variation in susceptibility to IgA nephropathy and to disease progression. Potentially, identifying and understanding genetic variation would provide insight into the aetiology of the condition, refine the prognosis in individual patients, and help to develop treatment strategies. In general, individuals with IgA nephropathy do not have affected family members. Therefore, the genetic contribution to IgA nephropathy will clearly be more complex to unravel than high penetrance monogenic traits. But this certainly does not preclude the possibility that the approach is feasible and will be useful. An encouraging precedent is inflammatory bowel disease, where an important contribution from variation in the NOD2/CARD15 gene has emerged from human genetic studies [2].

\section{Prevalence and Recurrence Risk}

Perhaps surprisingly, prevalence of IgA nephropathy is uncertain. This is because IgA nephropathy can only be diagnosed by renal biopsy. In gaining insight into the disease process, it would be valuable to know the frequency of deposition of IgA in the mesangium in humans, how commonly this is associated with evidence of nephropathy, and how often it leads to progressive renal impairment. Particularly valuable for guiding genetic studies would be to know how common each of these is in rela-
Patrick H. Maxwell, MD

Renal Section, Hammersmith Hospital, Imperial College

Du Cane Road

London W12 0NN (UK)

Tel. +44 208383 8594, Fax +44 208383 2062, E-Mail p.maxwell@imperial.ac.uk 
tives of individuals with IgA nephropathy compared to the general population (the recurrence risk). However, renal biopsy is an invasive procedure and carries a significant risk, so it is performed on relatively few people. Consequently, we have very limited information as to the true prevalence of IgA nephropathy in the general population. What is clear is that the perceived incidence of $\operatorname{Ig} \mathrm{A}$ nephropathy varies markedly depending on referral practices and biopsy criteria.

Perhaps our best estimates of the underlying prevalence of IgA deposition in the mesangium is the frequency of IgA deposits in series of postmortems and renal allografts. This suggests that mesangial IgA deposition occurs in $3-30 \%$ of unselected populations [3, 4]. At first glance this is surprising, since it is approximately two orders of magnitude more common than the incidence of renal disease attributed to IgA nephropathy. However, it would be consistent with the frequency of microscopic haematuria in the general population, given that renal biopsy in individuals with isolated microscopic haematuria shows mesangial IgA deposits in $~ 50 \%$.

Given this background, there is considerable uncertainty over the extent of the genetic contribution to IgA nephropathy. Several observations would be consistent with an important genetic contribution. First, there appear to be racial differences in susceptibility, which almost certainly exceed any differences attributable to patterns of nephrological practice. Thus IgA nephropathy appears more common in Chinese and Japanese populations, and rare in Africans. Second, there are a substantial number of multiply affected families, including reports from the USA, Italy and Australian Aborigines, and some of these would be consistent with autosomal-dominant transmission [5-7]. Third, relatives of individuals with IgA nephropathy have been reported to have urinary abnormalities, and increased circulating IgA relative to the general population [8]. Taken together, these observations argue strongly for an important genetic contribution to IgA nephropathy. As with other polygenic conditions, it is likely that interactions with the environment, epigenetic mechanisms and epistatic interactions between genes will all contribute to disease susceptibility.

\section{Strategies to Investigate the Genetic Contribution to IgA Nephropathy}

Investigation of genetic predisposition can use methods based on linkage to genetic variants, or association. In linkage-based approaches, related individuals are studied for sharing a trait, and a region of the genome. Initially the region that is implicated will usually be large, and identifying the mutation that lies within this region often represents a major challenge. Linkage-based approaches are very powerful, but require identification of multiply affected kindreds.

The linkage-based approach that has yielded tantalising results in IgA nephropathy has been to collect rare families where the condition seems to be inherited as a dominant trait, suggesting that a major gene underlies susceptibility in these families. Provided enough individuals whose disease status is clear are present in a pedigree it is straightforward to determine which regions of the genome co-segregate with the disease. In some cases where the number of cases in individual families are too small, power may be increased by pooling families. However, this will only be appropriate if the same locus underlies the disease in different families, which is often not the case. In a recent landmark study of 30 kindreds with IgA nephropathy it was shown that there was linkage to 6q2223 in $60 \%$ of the pedigrees studied [5]. The genetic evidence that this region is important in these kindreds is very strong indeed (lod score 5.6). This locus has been termed IGAN1, although as yet the causative mutation(s) in this region have not been identified. This illustrates the general point that when a region of the genome is implicated in a linkage study it is often a major challenge to identify the disease gene. An important question about $I G A N 1$ is whether the same region contributes to susceptibility to IgA nephropathy in the general population; as yet we have no insight into this at all.

A rather different approach to identify loci that contribute to polygenic diseases has been to identify affected sibling pairs, and then to test whether they share parts of the genome more often than would be predicted by chance. An advantage is that the method does not make assumptions about the way in which loci contribute to disease susceptibility, and can identify genes with less strong effects. This approach is based on the siblings sharing large regions of DNA, it is therefore 'linkage-based' and a positive result will implicate a block containing many genes, rather than pinpointing a disease-causing mutation. This has not yet been possible in IgA nephropathy because multiply affected sibships are uncommon.

Association-based approaches to investigate genetic contributions to disease are relatively easy to undertake, but are often hard to interpret as they can easily yield false-positive results. In principle, all that is required is to collect DNA from affected cases, and from a matched control population. The two groups are then genotyped at a specific locus to determine whether a genetic variant is observed at a different frequency in the case and control 
Table 1. Recently reported genetic associations with IgA nephropathy

\begin{tabular}{lllll}
\hline Gene & Location & $\begin{array}{l}\text { Relative } \\
\text { risk }\end{array}$ & Patients & Study \\
\hline Polymeric Ig receptor & 1q31-41 & 1.6 & 389 & {$[10]$} \\
L- and E-selectin & $1 \mathrm{q} 24-25$ & 1.9 & 346 & {$[11]$} \\
HLA-DRA & $6 \mathrm{p} 21.3$ & 1.9 & 313 & {$[12]$} \\
G protein $\beta_{3}$ subunit & $12 \mathrm{p} 13$ & 1.8 & 299 & {$[13]$} \\
Ig-mu-binding protein & $11 \mathrm{q} 13.2-13.4$ & 1.9 & 465 & {$[14]$} \\
TNF- $\alpha$ & $6 \mathrm{p} 21.3$ & 2.1 & 242 & {$[15]$} \\
IFN- $\gamma$ & $12 \mathrm{q} 14$ & 1.9 & 96 & {$[16]$} \\
Megsin & $18 \mathrm{q} 21.3$ & 2.1 & 423 & {$[17]$} \\
TGF- $\beta_{1}$ & $19 \mathrm{q} 13.1$ & 2.3 & 101 & {$[18]$} \\
\hline
\end{tabular}

populations using a $\chi^{2}$ test. A fairly large number of statistically significant associations with several different genes have been reported in IgA nephropathy. However, it is increasingly appreciated that there are many problems with this approach. Fundamental issues are the presence of stratification in the overall population with the consequence that the case and control populations may not be drawn from a genetically homogenous group, and the bias towards publishing positive results. As general principles, the likelihood of a finding from an association study proving to be correct are increased by the use of a sufficient size sample, the analysis / identification of biologically plausible genes, and evidence that the genetic variant causes a functional effect. Powerful evidence for a true association is provided by replication of the finding in other populations [9].

Some recently published studies of genetic association with IgA nephropathy are summarised in table 1 . In addition, other genetic variants have been reported to be associated with progressive IgA nephropathy, including variants in IL-10, IL-4, IL-1 receptor antagonist, CD14, T-cell receptor constant alpha chain, uteroglobin, plasminogen-activator inhibitor-1, angiotensinogen, angiotensin converting enzyme, aldosterone synthase, and nephrin. Although many of these genetic associations are plausible, they have not generally been observed in more than one study, and none of them can as yet be regarded to be proved to contribute to causing IgA nephropathy and/or contributing to its progression.

Two relatively new genetic approaches that have recently been applied to IgA nephropathy are family-based association studies and genome-wide association testing $[19,20]$. In the family-based approach, DNA is collected from the parents as well as affected individuals. The parental DNA allows the investigator to determine the alleles that an individual could have inherited. The cases and their parents (trios) can be genotyped at a candidate locus, to determine if the affected cases have inherited a particular variant more commonly than would have been predicted by chance. A problem with this approach is that it has limited power, since in interrogating a particular candidate locus only a proportion of trios will be informative [19]. Another issue is that DNA may not be available from the parents, but if siblings are available it may be possible to use their DNA to infer the parental genotypes. The major advantage of the family-based approach is that it eliminates the problem of population stratification inherent in association studies. In a recent study in China we used this approach to establish that genetic variation in Megsin is associated with IgA nephropathy [17]. Meg$\sin$ is a serine protease inhibitor expressed in mesangial cells, and has been shown to be over-expressed in biopsies from patients with IgA nephropathy.

Genome-wide association testing is another new approach to identifying genes underlying polygenic human diseases [20]. Although superficially similar to the genome scans that have been so successfully applied in linkage-based approaches, it is actually rather different. In linkage analysis a few hundred markers can be used to map the gene responsible for the disease in a family. When unrelated individuals are being studied any marker tested will only be in linkage disequilibrium with a small region of DNA, so to cover the genome a very large number of markers will be required. Since the extent of linkage disequilibrium varies from one part of the genome to another, we do not yet know how many markers would be required to perform the task effectively. The approach is clearly strengthened by using markers which are likely to be functional, since these may cause an effect in themselves as well as acting as a marker for a segment of DNA. Currently huge efforts are being made to identify sequence variants, and to determine the linkage disequilibrium structure of the genome in different populations. The International HapMap aims to define haplotype structure, which should greatly assist the search for genetic variants contributing to common diseases [21]. Once haplotypes are identified, the haplotype blocks across the genome will be identifiable with 'tagSNPs' making genome-wide association studies much more efficient.

An inherent aspect of a genome wide association study is that an enormous number of comparisons are made between genotypes in the affected and control samples, 
and appropriate statistical analysis is therefore potentially challenging. Estimates of the number of affected individuals that will be required to be successful are as yet imprecise - and inevitably based on a number of assumptions, but 1,000 affected individuals is generally considered to be a reasonable size for this approach. Crucially, technological advances in genotyping mean that the cost and amount of DNA required is falling rapidly. The current view is that whole genome association studies using tagSNPs are likely to be the most effective and practical approach to identifying common disease genes.

A general problem of genetic association studies is that population stratification between the cases and controls can produce false positive results, but the genome-wide approach does allow the extent of genetic heterogeneity to be assessed. An important advantage over other association approaches is that the approach is unprejudiced rather than based on testing candidate genes. Given our limited understanding of the proximal causes of IgA nephropathy and the mechanisms leading to its progression it is entirely plausible that any genetic variation in susceptibility occurs in genes that would not currently be considered as candidates. This approach has recently been applied to IgA nephropathy in Japan [14]. The study of $465 \mathrm{IgA}$ patients and 634 controls initially examined 88,148 gene-based SNPs in 94 of the patients to identify SNP variants potentially associated with disease. The entire collection was only genotyped for SNP's that showed a positive association in the initial segment. This approach identified 13 SNP's which were present at a different frequency in the patient and control populations with a $p$ value $<0.0001$. These included SNP's located in HLA-DRA and the immunoglobulin mu-binding protein, IGHMBP2. The latter protein may be involved in immunoglobulin class switching, so is a reasonable biological candidate. Although the result was highly statistically significant, it is important to appreciate that the relative risk was modest (odds ratio 1.85, CI 1.39-2.50). This illustrates the sensitivity of association-based methods for detecting moderate size effects. As yet, the finding has not (to our knowledge) been tested in other populations.

\section{Rodent Models of IgA Nephropathy and Genetics}

Research into the pathogenesis and treatment of human kidney disease in general has benefited substantially from rodent models. In the case of IgA nephropathy, marked differences in the IgA system between humans and rodents have limited this approach. From a genetic viewpoint analysis in model organisms has obvious ad- vantages, including the ability to generate specific genetic mutants, and defined breeding which facilitates genetic analysis and trait selection. Illustrating the mutational approach, mice lacking uteroglobin were found to develop deposition of IgA in the mesangium [22]. Rather disappointingly, it appears that the interaction between human IgA complexes and uteroglobin is different and there is not (as yet) clear evidence for a role of uteroglobin in human IgAN. Another mouse which develops IgA deposition and nephropathy is the ddY mouse. Onset is spontaneous, and varies in timing and severity. Illustrating how different this is from human IgAN, the mice do not develop haematuria. Nevertheless, a fascinating recent study suggests that genetic predisposition to human IgAN and nephropathy in ddY mice may overlap [23]. In this study, serial renal biopsies and genetic analyses were performed on several hundred ddY mice. Comparison of genetic markers in mice with early onset glomerular abnormalities compared to those without significant glomerular changes at 60 weeks implicated three different regions of the genome. Of these, one was very close to Sele (selectin) and another is syntenic to the human region encompassing IGAN1. These findings suggest that analysis of ddY mice may help to define the genetic basis of IgAN in humans, and that mice could provide a better model for aspects of the human disease than was previously supposed.

\section{Future Directions}

IgA nephropathy illustrates some important challenges about using genetic approaches to investigate human diseases in which the genetic contribution is complex. A particular issue is that identification of affected individuals requires an invasive procedure. As yet, genetic approaches have not provided decisive insights into this important condition. However, several powerful resources have been assembled around the world, and the methodology is advancing rapidly. Ultimately the success of the approach will depend on how large, and how complex, the genetic contribution is. How rapidly we answer this may depend on the extent of national and international collaboration that can be achieved. 


\section{References}

$\checkmark 1$ Donadio JV, Grande JP: IgA nephropathy. N Engl J Med 2002;347:738-748.

$\checkmark 2$ Hugot JP, Chamaillard M, Zouali H, Lesage S, Cezard JP, Belaiche J, et al: Association of NOD2 leucine-rich repeat variants with susceptibility to Crohn's disease. Nature 2001; 411:599-603.

>3 Waldherr R, Rambausek M, Duncker WD, Ritz E: Frequency of mesangial IgA deposits in a non-selected autopsy series. Nephrol Dial Transplant 1989;4:943-946.

-4 Suzuki K, Honda K, Tanabe K, Toma H, Nihei $\mathrm{H}$, Yamaguchi $\mathrm{Y}$ : Incidence of latent mesangial IgA deposition in renal allograft donors in Japan. Kidney Int 2003;63:2286-2294.

5 Gharavi AG, Yan Y, Scolari F, Schena FP, Frasca GM, Ghiggeri GM, et al: IgA nephropathy, the most common cause of glomerulonephritis, is linked to 6q22-23. Nat Genet 2000;26:354357.

6 O'Connell PJ, Ibels LS, Thomas MA, Harris M, Eckstein RP: Familial IgA nephropathy: a study of renal disease in an Australian aboriginal family. Aust NZ J Med 1987; 17:27-33.

7 Julian BA, Quiggins PA, Thompson JS, Woodford SY, Gleason K, Wyatt RJ: Familial IgA nephropathy: evidence of an inherited mechanism of disease. N Engl J Med 1985;312:202 208.

-8 Schena FP, Scivittaro V, Ranieri E, Sinico R, Benuzzi S, Di Cillo M, et al: Abnormalities of the IgA immune system in members of unrelated pedigrees from patients with IgA nephropathy. Clin Exp Immunol 1993;92:139144.

-9 Cardon LR, Bell JI: Association study designs for complex diseases. Nat Rev Genet 2001;2: 91-99.
10 Obara W, Iida A, Suzuki Y, Tanaka T, Akiyama F, Maeda $S$, et al: Association of singlenucleotide polymorphisms in the polymeric immunoglobulin receptor gene with immunoglobulin A nephropathy (IgAN) in Japanese patients. J Hum Genet 2003;48:293-299.

11 Takei T, Iida A, Nitta K, Tanaka T, Ohnishi Y, Yamada R, et al: Association between single-nucleotide polymorphisms in selectin genes and immunoglobulin A nephropathy. Am J Hum Genet 2002;70:781-786.

12 Akiyama F, Tanaka T, Yamada R, Ohnishi Y, Tsunoda T, Maeda S, et al: Single-nucleotide polymorphisms in the class II region of the major histocompatibility complex in Japanese patients with immunoglobulin A nephropathy. J Hum Genet 2002;47:532-538.

13 Thibaudin L, Berthoux P, Thibaudin D, Mariat $\mathrm{C}$, Berthoux $\mathrm{F}$ : $\mathrm{G}$ protein beta3 subunit C825T polymorphism in primary $\operatorname{IgA}$ nephropathy. Kidney Int 2004;66:322-328.

14 Ohtsubo S, Iida A, Nitta K, Tanaka T, Yamada $\mathrm{R}$, Ohnishi Y, et al: Association of a singlenucleotide polymorphism in the immunoglobulin mu-binding protein 2 gene with immunoglobulin A nephropathy. J Hum Genet 2005; 50:30-35.

15 Tuglular S, Berthoux P, Berthoux F: Polymorphisms of the tumour necrosis factor alpha gene at position -308 and TNFd microsatellite in primary IgA nephropathy. Nephrol Dial Transplant 2003;18:724-731.
16 Masutani K, Miyake K, Nakashima H, Hirano T, Kubo M, Hirakawa M, et al: Impact of interferon-gamma and interleukin-4 gene polymorphisms on development and progression of IgA nephropathy in Japanese patients. Am J Kidney Dis 2003;41:371-379.

17 Li YJ, Du Y, Li CX, Guo H, Leung JC, Lam MF, et al: Family-based association study showing that immunoglobulin A nephropathy is associated with the polymorphisms 2093C and $2180 \mathrm{~T}$ in the $3^{\prime}$ untranslated region of the Megsin gene. J Am Soc Nephrol 2004; 15: 1739-1743.

18 Carturan S, Roccatello D, Menegatti E, Di Simone D, Davit A, Piazza A, et al: Association between transforming growth factor beta 1 gene polymorphisms and IgA nephropathy. J Nephrol 2004; 17:786-793.

19 Cardon LR, Palmer LJ: Population stratification and spurious allelic association. Lancet 2003;361:598-604.

20 Wang WY, Barratt BJ, Clayton DG, Todd JA: Genome-wide association studies: Theoretical and practical concerns. Nat Rev Genet 2005; 6:109-118

21 The International HapMap Project. Nature 2003;426:789-796.

22 Zheng F, Kundu GC, Zhang Z, Ward J, DeMayo F, Mukherjee AB: Uteroglobin is essential in preventing immunoglobulin A nephropathy in mice. Nat Med 1999;5:1018-1025.

-23 Suzuki H, Suzuki Y, Yamanaka T, Hirose S, Nishimura H, Toei J, et al: Genome-wide scan in a novel IgA nephropathy model identifies a susceptibility locus on murine chromosome 10 , in a region syntenic to human IGAN1 on chromosome 6q22-23. J Am Soc Nephrol 2005; 16:1289-1299. 